Journal of Computer Science 4 (6): 427-436, 2008

ISSN 1549-3636

(C) 2008 Science Publications

\title{
Decreasing Overhead and Power Consuming in Ad-Hoc Networks by Proposal a Novel Routing Algorithm
}

\author{
A. Barati, A. Movaghar, H. Barati and A. Azizi Mazreah \\ Faculty of Engineering, Islamic Azad University-Dezful Branch, Iran
}

\begin{abstract}
An Ad-Hoc network has been constructed from a fabric of mobile nodes intercom connected by temporary wireless links. Nodes located beyond a single hop are reached using intermediate neighbors to forward messages over long distances. The problem with this method of communication is that mobility causes the network topology to be unstable. Traditional solutions maintain routes by exchanging information when ever the network topology changes. Using traditional solutions for maintaining routes in highly mobile environments creates high overhead cost which makes prohibition scalability. A new routing algorithm has been designed to support highly dynamic networks which maintain routs locally. The suggested algorithm in comparison with existing routing algorithms, not only minimize overhead, saving power considerably, but also improve scalability.
\end{abstract}

Key words: Wireless networks-Ad-Hoc networks-routing protocols-on demand routing-overhead

\section{INTRODUCTION}

Mobile Ad Hoc Network (MANET) is an efficient technology for providing a wide-area communication environment where installing the infrastructure of a wired network is difficult. It is also suitable for supporting communication among mobile nodes. For the last several decades, many routing protocols have been proposed to make the best use of wireless network technology ${ }^{[1,2]}$. As setting up base station or wired networks for mobile terminal is impossible or unfeasible use of these networks owing to simple configuration and low cost is very suitable. Some applications communicate in hostile environment without any central base station such as, navies in navy component, mobile computer meetings of people in areas where wired networks are not available, disaster recovery, management of emergencies (for instance in case of earth quake where all infrastructures are destroyed). On the other hand Ad-Hoc networks are a heterogeneous mix of different wireless and mobile devices, witch are supplied with battery and will be turn-of when battery is discharged ${ }^{[3]}$. So in this networks energy is very important issue and routing algorithm must be designed in a way that act accurately to use energy. Ad-Hoc routing algorithms can be categorized into two types Table driven and OnDemand. Table driven algorithms send periodical broadcasts to maintain a route Table. Algorithms classified as On-Demand construct routes only when they are needed ${ }^{[4]}$.
Dynamic Source Routing or $\mathrm{DSR}^{[5]}$ is an ondemand protocol which constructs routes as they are required. The path taken by a source routed message is determined by the originator and cannot be changed during transit. If the chosen path is broken, the sender must retransmit the message with an alternative route. The algorithm consists of two stages in route construction, route request and route maintenance ${ }^{[6]}$.

The Ad-Hoc On-demand Distance Vector or $\mathrm{AODV}^{[7]}$ is a combination of the DSDV and DSR algorithms described earlier. Routes are created by exchanging distance vectors on-demand.

Cluster Based Routing Protocols $(\mathrm{CBRP})^{[8]}$ partition the network into disjoint sets. The task of discovering routes is delegated to Cluster-heads which are elected because of their position and coverage. The aim of clustering is to reduce the number of packets flooded into the network by creating a temporary infrastructure.

The Optimized Link State Protocol (OLSP) ${ }^{[9]}$, Topology Dissemination Based Reverse Path $(\mathrm{TDBRP})^{[10]}$ and Zone Routing Protocol (ZRP) ${ }^{[11]}$ are derivatives of Link State Routing.

Associatively Based Routing (ABR) ${ }^{[12]}$ seeks to limit network traffic by discovering and using long lived routes between nodes which remain stable over time.

The rest of the study is organized as following: the proposed routing protocol is explained, evaluate the performance of DTRP and then compare other routing algorithms with DTRP.

Corresponding Author: A. Barati, Faculty of Engineering, Islamic Azad University-Dezful Branch, Iran 
Proposed routing protocol for AD-HOC networks: In this study we present a new solution for routing messages in mobile Ad-Hoc networks. The aim is to produce a simple and reliable routing algorithm which can tolerate a dynamic topology while minimizing routing overhead to conserve energy. Our proposed AdHoc routing algorithm uses localized maintenance to reduce the operational cost overhead. We refer to our algorithm as Demand Table Routing Protocol (DTRP). As the name suggests, the solution is demand driven, meaning that routes are created when they are needed. Routes are constructed by cost advertisement and confirmation. Localized maintenance ensures that routes stay intact as relocation changes the network topology.

Route representation: A route is represented by labeling the intermediate nodes on a path with a Route Pair containing the source and destination address. A set of nodes containing Route Pairs with the same source and destination addresses form a route linking the two endpoints together. In addition to the source and destination addresses, the Route Pair contains an Active flag and a timeout. A node will only forward an arriving message if it contains a Route Pair corresponding to the source and destination addresses of the packet. By forwarding, we mean to re-broadcast the message so that other nodes beyond the range of the source may receive it.

Route advertisment and creation: Nodes advertise themselves by sending periodic global Route Cost messages to inform all other nodes of the distance to the advertiser in hops. If multiple advertisements are received from the same source, lower costs take precedence. The nodes in Fig. 1 are labeled with their hop costs from an advertiser, A. To communicate with the advertiser, a node replies with a Route Create message.

The create message is propagated toward the advertiser by traversing intermediate nodes which have progressively lower costs. Depending on the distribution of nodes, there may be more than one pathway. Fig. 2 shows a route creation message following the least cost paths from C-A. The create message inserts a RoutePair entry into a Table at each node on the return path. A route pair entry contains: addresses of the two ends of a route, a timeout and an active status flag. Each node receiving the create message flags the route pair entry as active.

Route maintenance: Routes collapse when nodes move out of range and require maintenance to keep

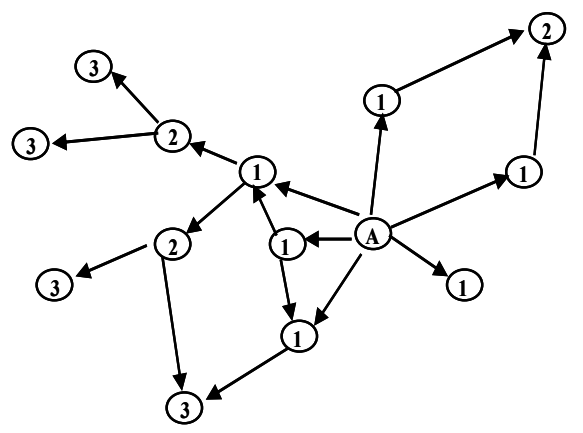

Fig. 1: Route advertisement

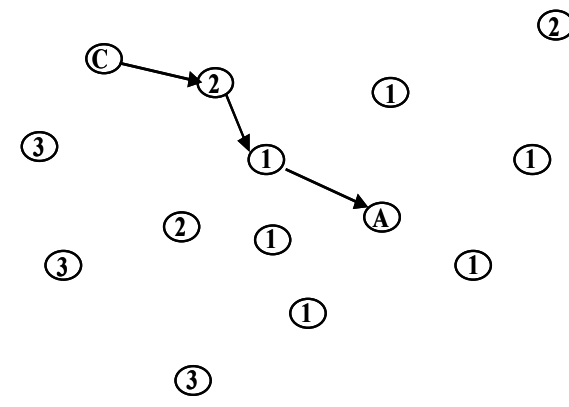

Fig. 2: Route creation

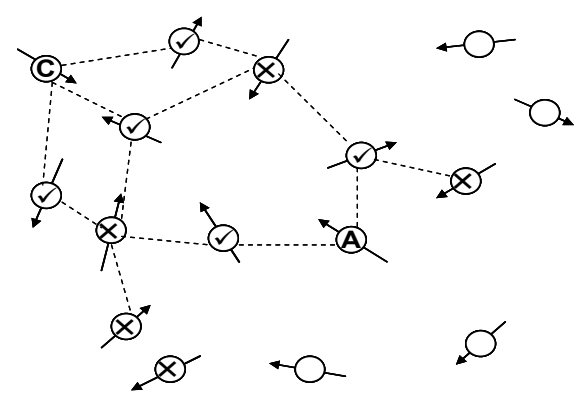

Fig. 3: Route maintenance before a topological change

them intact. This is accomplished by periodically exchanging a list of known active pairs in a Route Maintenance message between neighbors. On receiving a maintenance message, all of the new route pairs are stored as inactive entries. The active status of any previously known entries remains unchanged but their timeout periods are reset. Entries in the route pair Table are expired after a timeout period if they are not maintained. The initial state of a network is illustrated in Fig. 3. The nodes marked with a tick contain active route pairs for a path between $(\mathrm{A}, \mathrm{C})$. The nodes marked with a cross contain inactive route pairs for (A, C) which have been learned through localized maintenance. Fig. 4 illustrates the state of the 


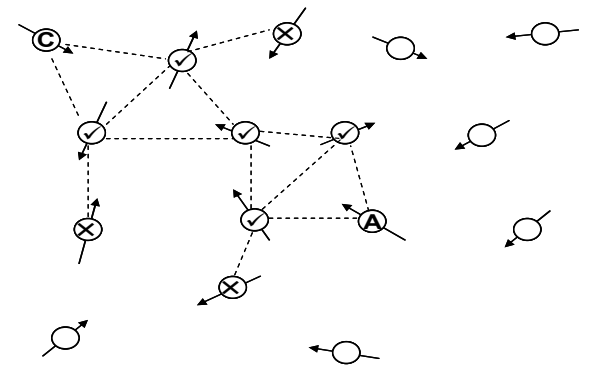

Fig. 4: Route maintenance after a topological change

network after the nodes have changed position. Some of the nodes have moved causing the chain to be broken, but have been replaced by nodes with inactive route pairs to keep the chain intact.

Route update frequance: For DTRP to operate efficiently, the frequency of route advertisements and maintenance messages need to be timed carefully. If sent too infrequently, the state of the network will change and the updates will contain information that is out of date. If sent too often, the network will become congested from the routing overhead. The ideal solution is to send advertisements at a frequency corresponding to the peak value of the distribution of nodes leaving range to captures as many nodes as possible before they become disconnected. Maintenance messages must be sent at a higher frequency to repair minor topological changes in-between the phases of route construction.

Reducing overhead: The time between periodic broadcasts are linked to the rate at which the topology of the network changes. The amount of global communication is reduced by making frequent localized repairs. This strategy aids in reducing the total overhead cost, thus saving energy. The aim of localized maintenance is to replace nodes that have moved out of range with nearby neighbors to keep routes intact. As one node leaves range, another arrives to replace it. Instead of incurring a worst case global route reconstruction cost each time a link is broken, there is only a single hop cost. This property causes the overhead cost of DTRP to scale with the number of broken links per route instead of $\mathrm{n}^{2}$ each time a link is broken, where $\mathrm{n}$ is the size of the network.

Duplicate messages: It is possible for many paths with equal distance to be created during route construction, causing duplication. Initially this appears to be inefficient but in fact works to our favor. By finding more than one pathway it is less likely that a route will become disconnected because of the inherent redundancy created by nodes with overlapping broadcast areas. Two problems arise when considering multi-path routes with DTRP: it is possible for a packet to be rebroadcast in the direction from which it came, or to be forwarded by multiple candidates in an overlapping area. To overcome both of these problems, packets headers are cached, forwarding only those which have not been seen before. The problem still exists that there may be more than one candidate available to forward the message creating duplicates. This can be avoided by inserting a short random delay before forwarding a packet. The delay introduces a race condition where the first node to forward the packet succeeds. Any duplicate packets queued pending transmissions by nodes within range are cancelled.

Header history cache: The aim of a Header History Cache (HHC) is to stop nodes from retransmitting the same message twice by remembering packet headers and discarding any duplicate messages. The HHC stops messages from backtracking in the direction from which they came and looping indefinitely. Packets are identified by their source address and a sequence number which is incremented by the source for each packet sent. The HHC also stores the total number of messages received when the entry was created and a hit count which is incremented each time the same packet is received. Entries are evicted from the cache when the ratio of hits/messages received falls below a threshold. The threshold can be adjusted depending on the size of the cache and the rate at which messages arrive. The eviction process is similar to a Least Recently Used (LRU) cache.

An example of the header history cache in operation: An example trace of hits/messages received against time is shown in Fig. 5. The threshold is arbitrarily set to evict entries when the ratio of hits/messages received drops below 0.25 for illustrative purposes. A new message is received each second. The trace initially has a value of 1.0 because a new packet header has just been inserted into the HHC. Three subsequent duplicates are received after 3, 4 and 11 seconds. The entry is evicted from the cache after 16 seconds when the trace drops below the threshold.

Evaluation criteria: Ad-Hoc networks allow direct communication between nodes using radio broadcasts ${ }^{[14,15]}$. As the nodes are mobile, there is a limited supply of power available to each device. The aims of an Ad-Hoc routing algorithm are to route messages efficiently with respect to path length and power consumption. These are the criteria that will use 


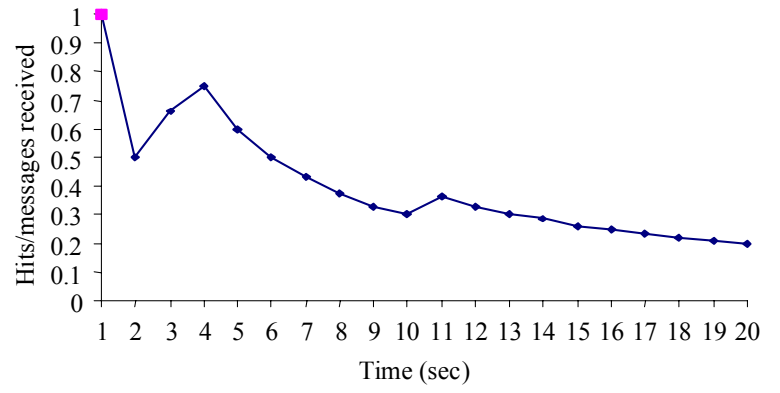

Fig. 5: Example trace for an entry in the header history cache

to evaluate the performance of ad-hoc networks in the rest of this study.

Path length: When all of the link costs are known, the router can find the cheapest path from source to destination. Assigning all links with a cost of one unit causes the router to find the shortest path. Cost based routing relies upon a stable network topology. Mobile Ad-Hoc networks cannot guarantee long lived point-topoint links. Under these circumstances, finding low cost paths by exchanging route information is a problem that cannot easily be solved. This is because the time needed to propagate total knowledge of the network can exceed the period of time for which the network remains stable. The aim is to find the shortest path between two nodes while consuming the least amount of power.

Power consumption: Mobile nodes are powered by batteries, therefore it is important to conserve energy in order to extend the lifespan of each device. This can be accomplished either by reducing the range of communication, or by reducing the total amount of data sent. These two options are mutually exclusive, requiring a cost/performance trade-off to be made. The broadcast range has a direct effect on the number of hops between source and destination. A shorter range implies a lower transmission cost but increases the number of hops needed to reach the destination. A longer range implies fewer hops but incurs a higher cost each time a message is forwarded. The frequency of route maintenance depends on the period of time for which nodes remain in contact with their neighbors. The network topology changes more often with a shorter range, creating a higher route overhead. Longer ranges allow route maintenance to be performed less frequently.

Metrics: The criteria for a good Ad-Hoc routing algorithm were identified in the previous section as minimization of power consumption by controlling the quantity and range of communication. In this study we define a set of metrics to quantify the performance of different Ad-Hoc networks against the criteria that has been decided upon. The metrics provide a method for objectively comparing different solutions.

Bandwith and latency: The performance of a network is traditionally measured using bandwidth and latency. The bandwidth of a link is the quantity of data that can be placed onto the link in a unit of time measured in bits per second (bps). Latency is the time needed for a signal to travel across the distance of a link medium. With respect to ad hoc networks latency indicates the time needed for a message to be propagated from source to destination including the time spent in queues at intermediate nodes. Bandwidth is defined by the medium and the limitations of the communication device being used. Of particular interest is how well the available bandwidth is utilized: the actual throughput vs. the theoretical maximum.

Energy: The simplest power-attenuation model suggests that signal strength falls at a rate of $1 / \mathrm{r}^{\mathrm{k}}$, where $r$ is the distance from the transmitting antenna and $\mathrm{k}$ is a value between 2 and 4, depending on range and interference in the environment. For our thesis, the precise value of $\mathrm{k}$ is irrelevant as long as there is attenuation, here we choose $\mathrm{k}=2$.

The attenuation model suggests that the energy required to transmit a signal is proportional to the distance it is being sent. More specifically, the energy needed to transmit a message of length 1 bytes over a distance $r$ meters at a cost of $b$ joules per byte, is $l b r^{k}$.

The costs of $b$ and $k$ are specific to each type of wireless device. Substituting $\mathrm{b}$ with 1 joule per byte gives the energy metric: $\operatorname{lr}^{2}$. These substitutions allow us to measure the energy consumed when sending the same message over variable distances. This assumes the properties of the underlying radio technologies are the same. The total energy, e, consumed by an ad-hoc network can be calculated as:

$$
\mathrm{e}=\sum \operatorname{lr}^{2}
$$

Routing overhead: Routing overhead is the amount of additional data that needs to be sent in order to create a route. The overhead is composed of advertisement and maintenance messages sent explicitly to create routes. The overhead o is the fraction of messages created by the routing algorithm out of the total amount of data sent: 


$$
o=\frac{1_{r}}{1_{t}}
$$

where $1_{r}$ the quantity of data is sent by the routing algorithm and $l_{t}$ is the total amount of data sent. A network with a lower routing overhead generates less traffic and is therefore more efficient with respect to the energy consumed. As $\mathrm{o} \rightarrow 1$ the routing algorithm consumes more of the network traffic which is undesirable.

Performance evaluation of DTRP: In order to evaluate performance of DTRP, NS2 simulator was used. Enough bandwidth is available for each node to transmit up to 100 packets per second, with a Maximum Transmission Unit (MTU) is one kilobyte. Messages larger than the MTU are fragmented prior to sending by the sliding window protocol and reassembled by the receiver. The sliding window protocol also permits out of order delivery. The position of each node is recomputed at a frequency of $100 \mathrm{~Hz}$ to accurately represent the highly dynamic connectivity of an AdHoc network.

Flooding a mobile networek: In this experiment, we enable node mobility and the DTRP route maintenance scheme. The aim is to show that DTRP is more energy efficient than broadcasting in a mobile network and that altering connectivity affects performance for identical work loads.

Methods: Nodes were allowed to roam and communicate in two differently shaped arenas. The first arena was a square $10 \mathrm{~m} \times 10 \mathrm{~m}$, the second a rectangle $20 \mathrm{~m} \times 5 \mathrm{~m}$. Both experiments used 25 nodes with evenly distributed start positions. A broadcast range of $3 \mathrm{~m}$ was selected to ensure sufficient connectivity between nodes. A stationary node was positioned at the centre of each arena to act as the receiver. All other nodes walked randomly within the arena at a maximum velocity of $0.7 \mathrm{~m} \mathrm{sec}^{-1}$, sending $1000 \times 512$ byte packets to the receiver. The experiment was done once with full network broadcasting using the square arena and then using DTRP in the square and rectangle arenas.

Maintenance messages: Established cost based routes collapse if the network topology becomes unstable due to motion. Maintenance ensures that routes stay intact. Regular localized maintenance messages are sent by DTRP to inform neighboring nodes of active routes in their vicinity. The frequency of maintenance messages depends on the stability of the network.

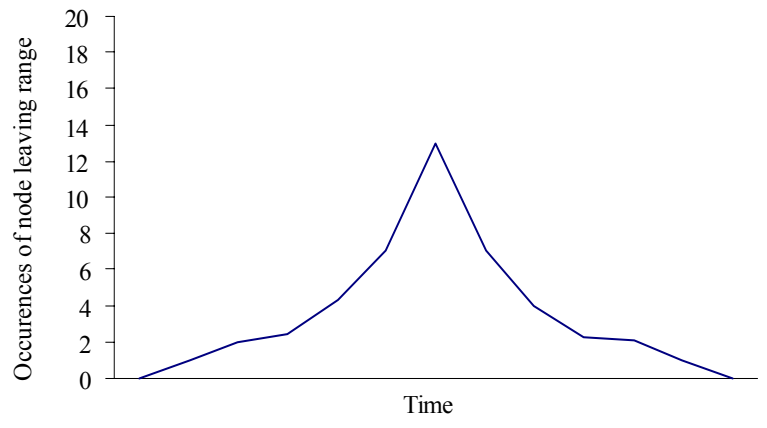

Fig. 6: Nodes leaving range over $10 \mathrm{~min}$ 's for 25 nodes with range of $3 \mathrm{~m}$

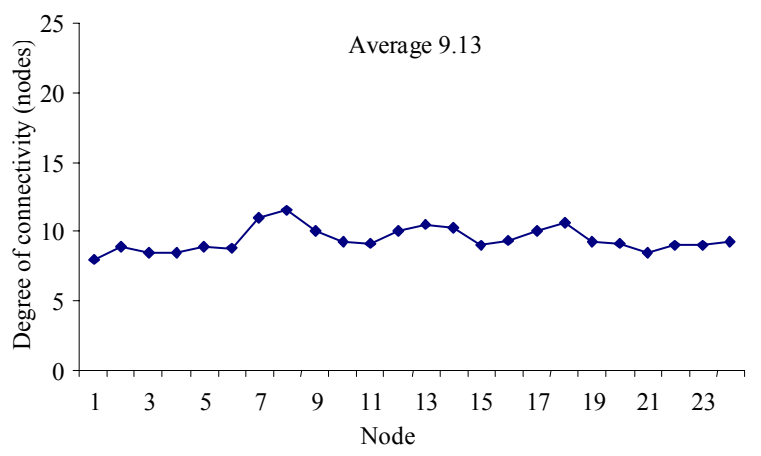

Fig. 7: Degree of Connectivity for mobile nodes in square arena

DTRP algorithm parameters: The frequency of advertisements and maintenance messages has to be determined for optimal results. The distribution of nodes leaving range was measured in a test experiment over a period of $10 \mathrm{~min}$. The results are shown in Fig. 6 Nodes leave range most often after 4.4 seconds, suggesting an advertisement frequency of 4 seconds and maintenance frequency of 2 seconds is sufficient.

Results: Fig. 7 and Fig. 8 show the degree of connectivity and path lengths from each node to the receiver for the network confined to a square arena. The average path length from each node to the receiver is very similar for all nodes while the degree of connectivity is spread over a wide range. Fig. 9 and Fig. 10 show the average path lengths and degree of connectivity for nodes in the network confined to a rectangle arena. The nodes appear to have similar degrees of connectivity with a greater variation in path lengths to the receiver. Fig. 11 shows that a $13 \%$ energy saving was achieved using DTRP over broadcasting in the square network, with a route overhead of only $0.9 \%$. Changing the arena to a rectangle produced a 


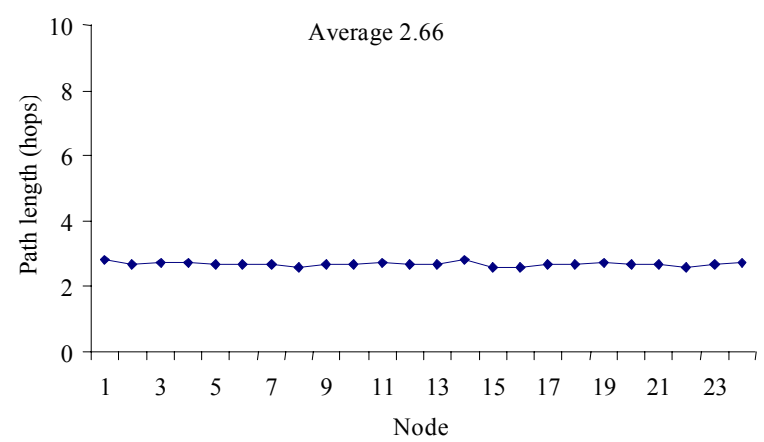

Fig. 8: Path lengths for mobile nodes in square arena

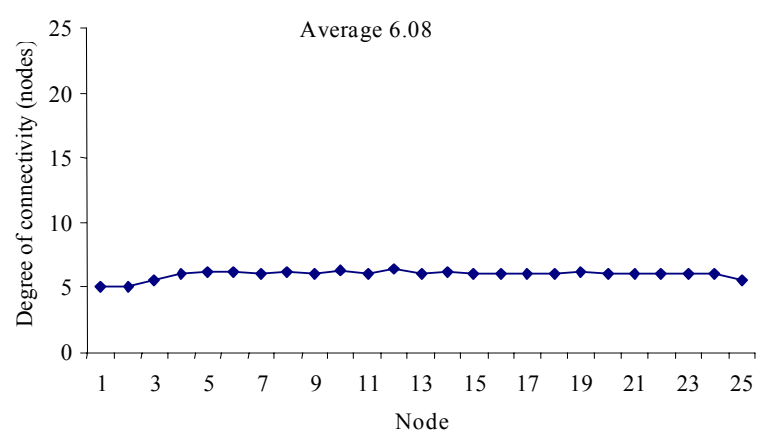

Fig. 9: Degree of connectivity for mobile nodes in rectangle arena

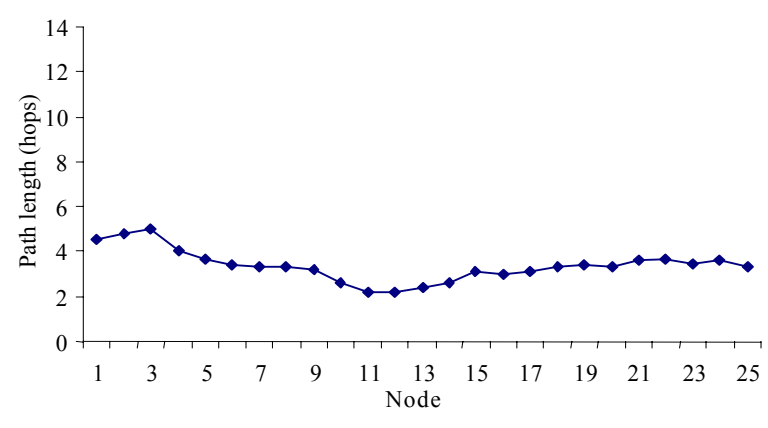

Fig. 10: Path Lengths for mobile nodes in rectangle arena

$25 \%$ improvement over broadcasting, with a $3.2 \%$ route overhead for the same workload.

Client/server application: The work loads and motion characteristics evaluated in the previous sections indicate the performance of DTRP under heavy load with random motion. In this experiment a work load representing bursty client/server traffic is combined with the motion characteristics of pedestrians to increase the fidelity of the simulated network. A larger

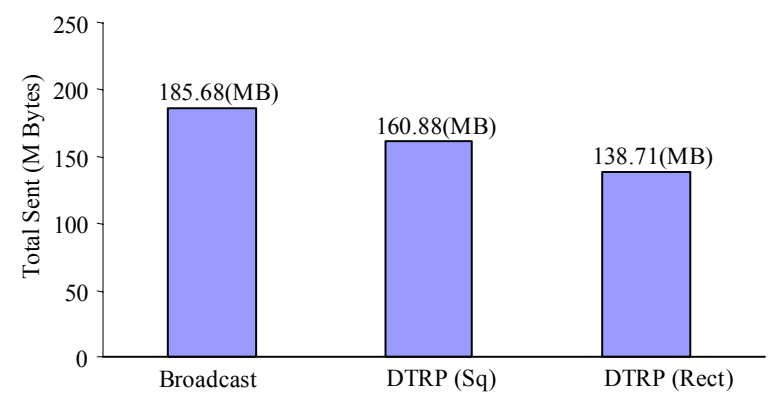

Fig. 11: Total bytes sent in broadcast and DTRP networks with motion

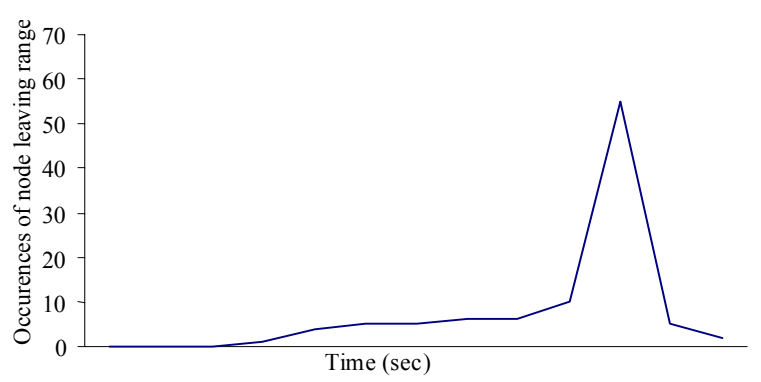

Fig. 12: Nodes leaving range over $10 \mathrm{~min}$ : 50 nodes with range $5 \mathrm{~m}$

network is used to create longer routes which better demonstrates the efficiency of DTRP.

Method: A road $50 \mathrm{~m}$ long by $10 \mathrm{~m}$ wide was populated with 50 nodes. One of the nodes was placed in the center of the road to act as a server which remained stationary. The remaining 49 client nodes sent 10 requests of 128 bytes to the server at random time intervals while in motion. The server responded by randomly selecting one of the queued requests and replying with 4 Kbytes of data.

Motion: To create realistic pedestrian motion the steering behavior selects a random target at one end of the road. The trajectory is recomputed by offsetting the target by a small amount along the edge of the road.

DTRP algorithm parameters: For this experiment the range was set at $5 \mathrm{~m}$ and the maximum node velocity was limited to $0.7 \mathrm{~m} / \mathrm{sec}$. Fig. 12 shows the distribution of nodes leaving range over a period of $10 \mathrm{~min}$. The longest period of time a node stays in range is 7.2 seconds. Using this data, the advertisement frequency was set to 7 seconds with maintenance messages being sent every 3.5 seconds. 


\section{RESULTS AND DISCUSSION}

The average path lengths and degrees of connectivity are shown in Fig. 13 and Fig. 14. The average path length was 4.36 hops and the average degree of connectivity was 7.47 nodes. Fig. 15 shows a $31 \%$ energy saving was achieved using DTRP over broadcasting, which is a $20 \%$ improvement over the energy saving in with 25 nodes. The improvement was due to the increase in average path length which allowed more focused routes to form.

Trading energy for bandwidth: Another experiment was done with different ranges to show the effect on throughput. The experiment used 30 nodes in a $30 \mathrm{~m} \times$ $10 \mathrm{~m}$ arena with broadcast ranges between $5 \mathrm{~m}$ and $10 \mathrm{~m}$. The average time between a client sending a request and receiving a response as the range was increased. Each node sent 20 requests at random intervals. Advertisements were sent every $5 \mathrm{sec}$. and maintenance messages every 2.5 seconds. Fig. 16 and Fig. 17 show the average degree of connectivity and path length for the same network while increasing the range.

The total energy consumed and average service time is plotted in Fig. 18 and Fig. 19. The average service time is the average time between clients sending a request and receiving a response.

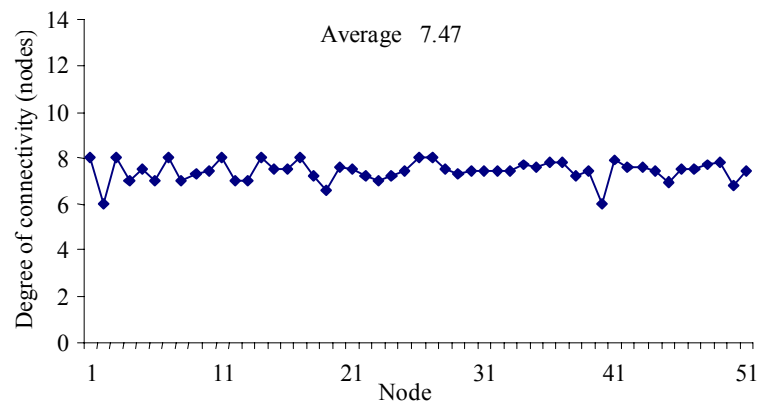

Fig. 13: Degrees for 50 mobile nodes

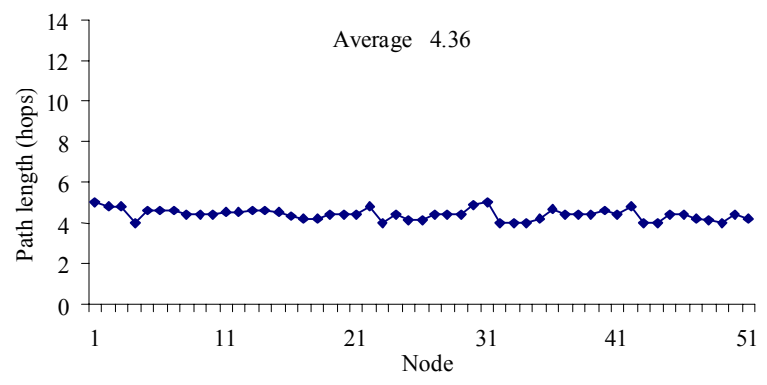

Fig. 14: Path Lengths for 50 mobile nodes
Increasing the range causes the total amount of data sent and average service time to fall. The maximum throughput is achieved with a range of $9 \mathrm{~m}$, after which the network becomes congested causing energy and service time to increase sharply. The results show that energy can be traded for throughput by adjusting range. Least energy is consumed with the shortest range.

Comparison with other approaches: The aim of this section is to compare the efficiency and scalability of DTRP with exist routing algorithms. In this section we'll compare overhead and energy consuming in DTRP with other algorithms.

Method: Each of the algorithms were analyzed to calculate the route overhead ${ }^{[13]}$ taking into account the network size, number of routes and average path length. The analysis is based on the probability of route failure which was calculated by measuring the number of broken links and average path length from simulation data.

Probability of a route failure: For our analysis, we need to know how often a route is broken. The probability of a broken route $\mathrm{P}_{\mathrm{r}}$ can be determined from the number of broken links, $b$ and the average path length, $\mathrm{k}$ over the period of time for a node to send $t$ messages.

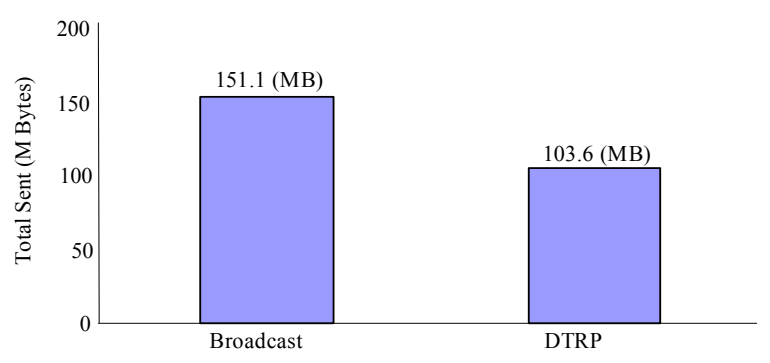

Fig. 15: Total bytes sent in client/server network with 50 nodes

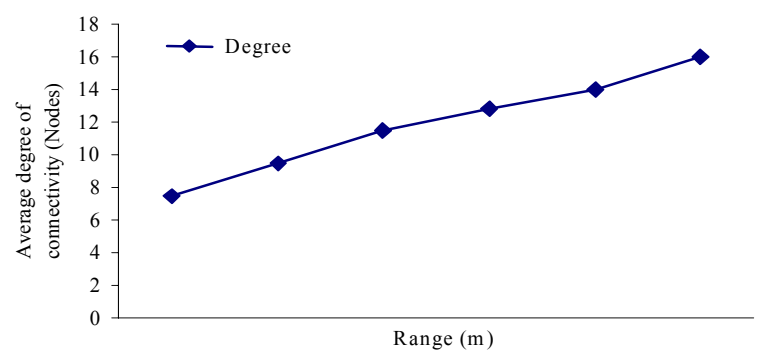

Fig. 16: Average Degrees with varying ranges 


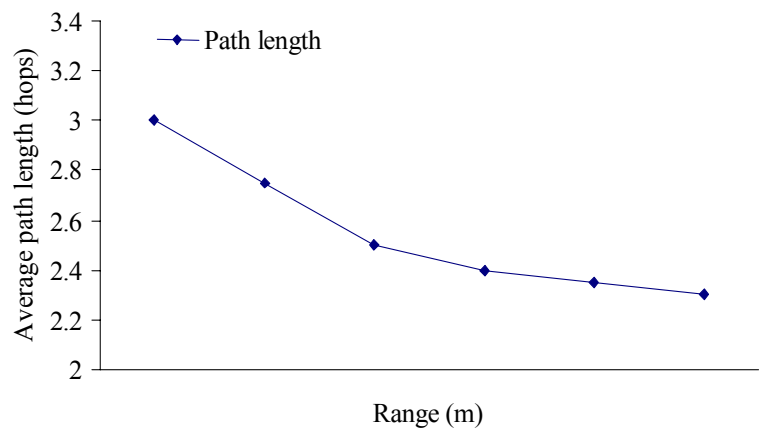

Fig. 17: Path lengths with varying ranges

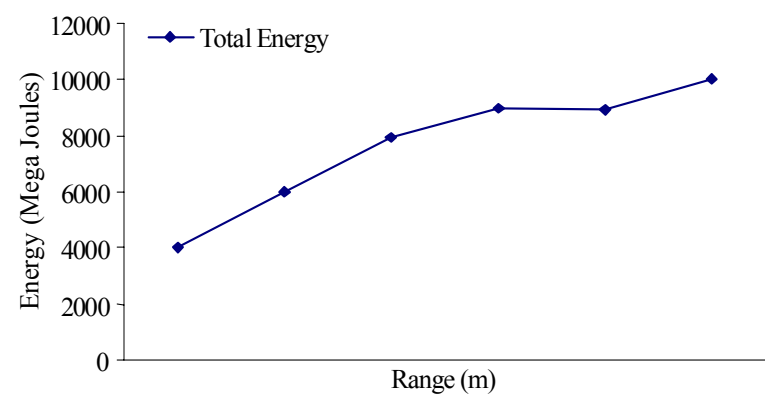

Fig. 18: Total energy consumed

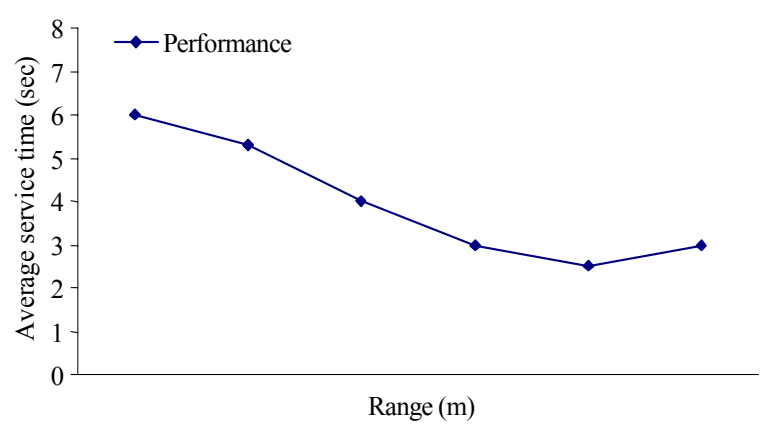

Fig. 19: Performance

The probability of a broken link per message is:

$$
P_{r}=b / t
$$

The probability of a broken route per message is derived from the probability of a broken link:

$$
\mathrm{P}_{\mathrm{r}}=1-\left(1-\mathrm{P}_{\mathrm{b}}\right)^{\mathrm{k}}
$$

Calculating overhead: The algorithms were analyzed to identify their characteristics and deduce how costly they are. In this section we state the how the cost of each algorithm was calculated.
DSDV: The DSDV algorithm has a static advertisement cost for periodic distance vector broadcasts from each node and a dynamic cost which is incurred each time a link is broken:

$$
\begin{aligned}
& \text { Static cost }=(\text { nodes })^{2} \times \text { frequency } \\
& \text { Dynamic cost }=\left(\mathrm{P}_{\mathrm{r}} \times \mathrm{t}\right) \times \text { routes } \times \text { nodes } \\
& \text { Total cost }=\text { Static cost }+ \text { Dynamic cost }
\end{aligned}
$$

DSR: Routes are created by fully broadcasting an advertisement to all nodes followed by a reply along the shortest path to acknowledge the route. Maintenance messages are sent each time a route fails, causing the route creation process to be repeated.

$$
\begin{aligned}
& \text { Route create }=(\text { nodes } \times \text { routes })+(\text { route } \times k) \\
& \text { Route maintenance }=\left(\mathrm{P}_{\mathrm{r}} \times \mathrm{t}\right) \times \text { Route create } \\
& \text { Total cost }=\text { Route create }+ \text { Route maintenance }
\end{aligned}
$$

AODV: AODV has the creation cost of DSR and the maintenance cost of DSDV. Nodes participating in routing send periodic updates to their neighbors. We assume that all nodes participate in at least one route.

$$
\begin{aligned}
& \text { Updates }=\text { frequency } \times \text { nodes } \\
& \text { Route create }=(\text { node } \times \text { routes })+(\text { routes } \times k) \\
& \text { Route maintenance }=\left(\mathrm{P}_{\mathrm{r}} \times \mathrm{t}\right) \times \text { routes } \times \text { nodes } \\
& \text { Total cost }=\begin{array}{c}
\text { Updates }+ \text { Route create }+ \text { Route } \\
\text { aintenance }
\end{array}
\end{aligned}
$$

TORA: TORA has the same route creation cost as DSR. On route failure, the maintenance cost is one traversal of the average path length to repair the broken link by readjusting heights.

$$
\begin{aligned}
& \text { Route create }=(\text { nodes } \times \text { routes })+(\text { routes } \times k) \\
& \text { Route maintenance }=\left(P_{r} \times t\right) \times k \times \text { routes } \\
& \text { Total cost }=\text { Route create }+ \text { Route maintenance }
\end{aligned}
$$

DTRP: Nodes periodically send global cost advertisements if they offer a service. A route is constructed by replying to an advertisement with a creation message. Localized maintenance messages are sent periodically to keep route pairs fresh. The 
Table 1: for a square network with random walks

\begin{tabular}{llll}
\hline Nodes & $\mathrm{k}$ & $\mathrm{b}$ & $\mathrm{P}_{\mathrm{r}}$ \\
\hline 25 & 1.626777 & 496.08 & 0.013415 \\
36 & 1.855285 & 593.89 & 0.018286 \\
49 & 2.179872 & 626.22 & 0.022719 \\
64 & 2.426383 & 682.53 & 0.027378 \\
81 & 2.699931 & 694 & 0.030923 \\
100 & 2.992618 & 733.72 & 0.036152 \\
\hline
\end{tabular}

Table 2: Simulation data for a rectangle network with random walks

\begin{tabular}{llll}
\hline Nodes & $\mathrm{k}$ & $\mathrm{b}$ & $\mathrm{P}_{\mathrm{r}}$ \\
\hline 30 & 2.123489 & 486.66 & 0.017145 \\
40 & 2.755818 & 508.75 & 0.0231193 \\
50 & 3.363172 & 454.72 & 0.025261 \\
60 & 3.946521 & 527.83 & 0.034271 \\
70 & 4.54326 & 577.77 & 0.043009 \\
80 & 5.200739 & 635.8 & 0.053898 \\
90 & 5.80658 & 468.27 & 0.044476 \\
100 & 6.466557 & 453.12 & 0.047839 \\
\hline
\end{tabular}

frequency of advertisements and maintenance messages depend on the length of time nodes stay in range.

$$
\begin{gathered}
\text { Advertise }=\text { nodes } \times \text { Advertise Frequency } \\
\text { Route create }=(\text { routes } \times \mathrm{k}) \times \text { Advertise } \\
\text { Frequency }
\end{gathered}
$$$$
\text { Route maintenance }=\text { nodes } \times \text { Maintenance }
$$$$
\text { Frequency }
$$

Total cost $=$ Advertise + Route create + Route Maintenance

Comparing scalability by size of network: Three different types of motion characteristics were evaluated for networks with sizes between 25 and 100 nodes. In each case we assumed that routes were created to a single static node as in the previous chapter. For each of the networks we recorded the number of broken links, $b$ and average path length, k over a period of $10 \mathrm{~min}$. The probability of a broken route, $\mathrm{P}_{\mathrm{r}}$ was calculated from the values of $k$ and $b$. The measurements and calculated probabilities are presented in Table 1 and Table 2. A broadcast frequency of $1 \mathrm{~Hz}$ was used to calculate the cost of DSDV and AODV. The cost of the DTRP algorithm was calculated with advertisements sent every 4 seconds and maintenance messages sent every 2 sec. The routing costs for each algorithm are presented in Table 3 and plotted in Fig. 20 using a log scale.

Compare consumed energy through node speed: This experiment compares energy consuming in different routing algorithms with different node speed. Simulated information was recorded for a network consists of 50 nodes with speed between $1 \mathrm{~m} \mathrm{sec}^{-1}$ to 8
Table 3: Route overhead in thousands of packets

\begin{tabular}{lllllll}
\hline Nodes & Networks capacity & DSDV & DSR & AODV & TORA & DTRP \\
\hline 30 & 1800 & 1435 & 959 & 914 & 64 & 23 \\
40 & 2400 & 3131 & 2322 & 2197 & 151 & 34 \\
50 & 3000 & 5213 & 3966 & 3746 & 252 & 47 \\
60 & 3600 & 9439 & 7766 & 7319 & 483 & 62 \\
70 & 4200 & 15404 & 13278 & 12511 & 814 & 79 \\
80 & 4800 & 24278 & 21773 & 20493 & 1335 & 98 \\
90 & 5400 & 26235 & 22763 & 21437 & 1388 & 118 \\
100 & 6000 & 34416 & 30364 & 28487 & 1848 & 141 \\
\hline
\end{tabular}

Table 4: Power consumption correspond to increase speeds

\begin{tabular}{llllll}
\hline Node speed & DSDV & DSR & AODV & TORA & DTRP \\
\hline 1 & 17324 & 16817 & 16751 & 10211 & 4164 \\
2 & 18821 & 18195 & 18156 & 12048 & 5041 \\
3 & 20837 & 20381 & 20345 & 13429 & 5518 \\
4 & 21954 & 21430 & 21425 & 14529 & 6371 \\
5 & 23318 & 22962 & 22951 & 15819 & 7628 \\
6 & 23814 & 23501 & 23494 & 16527 & 8649 \\
7 & 23584 & 23396 & 23311 & 16221 & 9260 \\
8 & 24451 & 24224 & 24208 & 17142 & 10041 \\
\hline
\end{tabular}

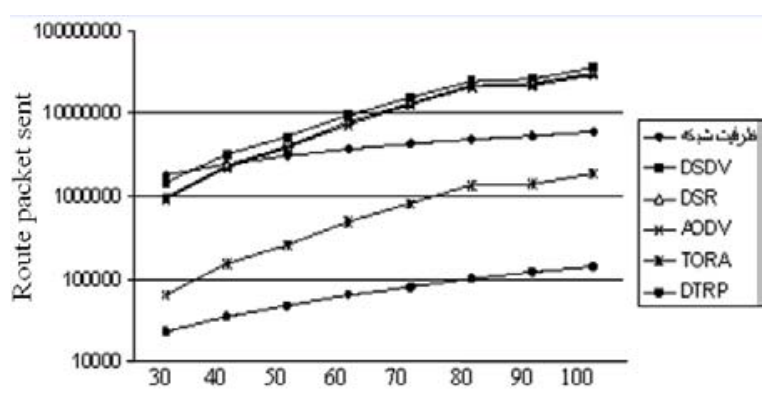

Fig. 20: Comparison of routing overhead for increasingly large network

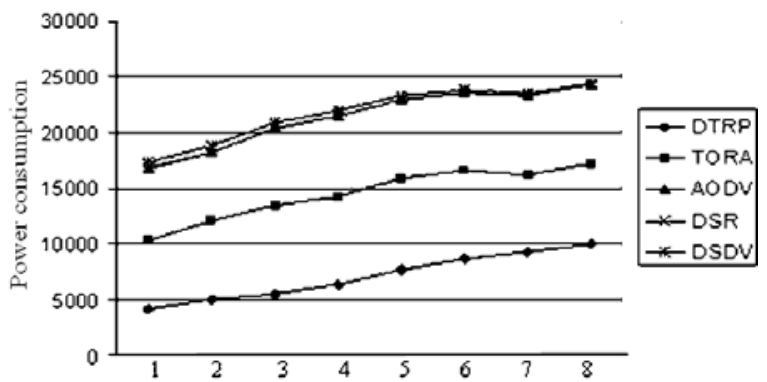

Fig. 21: Comparing power consumption in DTRP with other algorithms

$\mathrm{m} \mathrm{sec}{ }^{-1}$. The number of broken links increases with node speed while the average path length remains constant. The measurements have been shown in Table 4 and plotted on a log scale in Fig. 21.

The results show that DTRP sends the least number of route packets. It is also noted that for a network with more than 40 nodes DSDV, DSR and AODV need to send more packets than the available bandwidth. 


\section{CONCLUSION}

In this research, a new routing algorithm has been proposed for Ad-Hoc networks. In this method the route is created by labeling middle nodes and will create a chain between source and destination. As the network is mobile, a chain does not stay intact for long. Frequent localized maintenance messages repair the breakages caused by minor topological changes at low cost. The savings in cost overhead are derived from selective route cost advertisements and localized maintenance which limits the amount of data flooded onto the network.

To evaluate DTRP we used NS-2 simulation and a comparative analysis was made using experimental data to show that our solution has a significantly lower cost overhead than existing routing algorithms for highly mobile Ad-Hoc networks. The benefits of our solution are lower power consumption and increased scalability.

\section{REFRENCES}

1. Hong, X., K. Xu and M. Gerla, 2002. Scalable routing protocols for mobile Ad Hoc networks. IEEE Network, 16: 11-21. DOI: 10.1109/MNET. 2002.1020231.

2. Royer, E.M. and C.K. Toh, 1999. A review of current routing protocols for Ad-Hoc mobile wireless networks. Personal Commun., IEEE, 6: 46-55. DOI: 10.1109/98.760423.

3. Feeney, L.M. and M. Nilsson, 2001. Investigating the energy consumption of a wireless network interface in an Ad-Hoc networking environment. In: Proceeding IEEE INFOCOM, Anchorage, AK, USA, 3: 1548-1557.

4. Jayakumar, G. and G. Ganapathy, 2007: Performance comparison of mobile Ad-Hoc network routing protocol. Int. J. Comput. Sci. Network Security, 7: 77-84.

5. Johnson, D. and D. Maltz, 1996. Dynamic source routing in Ad-Hoc wireless networks. Mobile Comput., 323: 153-181.

6. Barati, A. A. Movaghar, M.R. Eslami Nejad and A. Bazrgar, 2007. Presenting an On-Demand Routing Algorithm for Wireless Mobile Ad-Hoc Networks, Proceedings of 12th Annual Int. CSI Computer Conference, Tehran, Iran, pp. 656-663, January 2007 (in Persian).
7. Perkins, C.E. Royer, E.M., 1999. Ad-hoc ondemand distance vector routing. Proceedings WMCSA Second IEEE Workshop on Mobile Computing Systems and Applications, '99. pp: 90100 DOI: 10.1109/MCSA.1999.749281, ISBN: 07695-0025-0

8. Krishna, P., N. Vaidya, M. Chatterjee and D.K. Pradhan, 1997. A cluster based approach for routing in dynamic networks. ACM Sigcomm Compu. Commun., 27: 49-64. ISSN: 0146-4833.

9. Jacquet, P., P. Muhlethaler, T. Clausen, A. Laouiti, A. Qayyum and L. Viennot, 2001. Multi topic conference, 2001. In: Proceeding in IEEE INMIC, 2001. pp: 62-68. DOI: 10.1109/INMIC.2001.995315.

10. Ogier, M., R. Templin and F. Lewis, 2004. Topology Dissemination Based on Reverse-Path Forwarding (TBRPF). In: Internet-Request for Comments 3684, IETF, http://www.ietf. org $/ \mathrm{rfc} / \mathrm{rfc} 3684$.txt.

11. Haas, Z. and M. Pearlman, 1998. The zone routing protocol for highly reconfigurable Ad-Hoc networks. In: Proceedings of ACM SIGCOMM 98, Vancouver, British, Columbia.

12. Toh, C., 1997. Associativity-based routing for AdHoc mobile networks. Wireless Personal Commun. J., 4: 103-139. DOI: 10.1023/A:1008812928561.

13. Clausen, T., P. Jacquet and L. Viennot, 2004. Analyzing control traffic overhead versus mobility and data traffic activity in mobile ad-hoc network protocols. ACM Wireless Networks J. (Winet), 10: 447-455. DOI: 10.1023/B:WINE.0000028548. 44719. fe.

14. Jayakumar, G. and G. Gopinath, 2007. Ad Hoc mobile wireless networks routing protocols-a review. J. Comput. Sci., 3: 574-582. ISSN: 15493636.

15. Pirzada, A.A., C. McDonald and A. Datta, 2006. Performance comparison of trust-based reactive routing protocols. IEEE Transact. Mob. Comput., 5: 695-710. DOI: 10.1109/TMC.2006.83. 